\title{
Resolving Name Conflicts for Mobile Apps in Twitter Posts
}

\author{
Sangaralingam Kajanan, Ahmed Shafeeq Bin Mohd Shariff, Kaushik Dutta, \\ and Anindya Datta \\ School of Computing, \\ National University of Singapore, Singapore \\ skajanan@comp.nus.edu.sg, ahmedshafeeqm@gmail.com, \\ \{dutta, datta\}@comp.nus.edu.sg
}

\begin{abstract}
The Twitter platform has emerged as a leading medium of conducting social commentary, where users remark upon all kinds of entities, events and occurrences. As a result, organizations are starting to mine twitter posts to unearth the knowledge encoded in such commentary. Mobile applications, commonly known as mobile apps, are the fastest growing consumer product segment in the history of human merchandizing, with over 600,000 apps on the Apple platform and over 350,000 on Android. A particularly interesting issue is to evaluate the popularity of specific mobile apps by analyzing the social conversation on them. Clearly, twitter posts related to apps are an important segment of this conversation and have been a main area of research for us. In this respect, one particularly important problem arises due to a name conflict of mobile app names and the names that are used to refer the mobile apps in twitter posts. In this paper, we present a strategy to reliably extract twitter posts that are related to specific apps, but discovering the contextual clues that enable effective filtering of irrelevant twitter posts is our concern. While our application is in the important space of mobile apps, our techniques are completely general and may be applied to any entity class. We have evaluated our approach against a popular Bayesian classifier and a commercial solution. We have demonstrated that our approach is significantly more accurate than both of these. These results as well as other theoretical and practical implications are discussed.
\end{abstract}

Keywords: Affinity, Microblogs, Twitter, Mobile Apps, Filter.

\section{Introduction}

The Twitter platform has emerged as a leading medium of conducting social commentary, where users remark upon all kinds of entities, events and occurrences. As a result, organizations are starting to mine twitter posts to unearth the knowledge encoded in such commentary. Applications that can benefit from such knowledge discovery are many: trending topic discovery, sentiment analysis of consumer products and gauging public reaction to political campaigns to name a few. A key requirement of a majority of such applications is the timely

A. Bhattacherjee and B. Fitzgerald (Eds.): Future of ICT Research, IFIP AICT 389, pp. 3- 17, 2012.

(c) IFIP International Federation for Information Processing 2012 
identification of twitter posts related to specific entities of interest, like products, persons or events. Such identification is well understood to be difficult due to a number of reasons, including (a) real-time discovery of relevant twitter posts given their massive rate of generation [1320, (b) handling multi-lingual posts and (c) interpreting highly cryptic tweets, driven by brevity constraints [7.

In this work, we will be exploring this problem, i.e., the real-time identification of microblog postings that contain references to pre-specified entities of interest. For example, we might wish to identify tweets that talk about the movie "Harry Potter and the Deathly Hallows: Part 2".

Two key problems that need to be addressed to perform such identification arise due to (a) the practice of aliasing entity names and (b) naming conflicts that arise between the entity of interest and other objects. Aliasing, driven by the need to conserve space, is the practice of using a subset of complete entity names (such as "Harry potter", for "Harry potter and the deathly hallows: Part $2 ")$ to refer to the entity. Clearly, if the identification system was unaware of such aliasing, it would perform poorly. The second problem, i.e., naming conflicts arise from semantic overloading of entity names, and is a common problem in the general search area. For instance, a film historian seeking information about the movie "ten commandments" (a phrase with wide connotations) will find that a simple search with just the movie title yields an enormous amount of information not related to the movie. However, adding contextual clues to the title (e.g., "ten commandments movie", "ten commandments de mille", "ten commandmentsheston") yield high quality results [11246]. In most cases (such as in regular internet search), the user performing the search is aware of additional context clues (such as the fact Charlton Heston played the lead role in Ten Commandments) and can easily expand the search term.

In Twitter, the aliasing and entity name conflict problems assume special significance as the brevity of twitter posts precludes the usage of traditional context clues. While this problem arises while searching any entity type, we ran into this issue particularly often in the domain of mobile applications, as we explain below.

Mobile applications, commonly known as mobile apps, are the consumer software for smart-devices, such as those running on Google's Android [10] or Apple's iOS 3] platforms. They represent the fastest growing consumer product segment in the history of human merchandizing 301716], with over 617,000 apps on the Apple platform and over 357,000 on Android. Their growth rate is astonishing, with nearly 3500 apps being added to the Android market and Apple app stores every day.

The importance of apps is underscored by the fact that the future of the mobile strategies of both Apple and Google are heavily dependent upon who wins the mobile app wars [342132]. With this backdrop, there is tremendous academic as well as commercial interest in mobile apps.

An interesting feature about mobile apps is their virality - most successful apps (e.g., Angry Bird, Talking Tom, Flashlight etc.) gain popularity not by explicit outbound marketing, but rather, through viral word-of-mouth spread. Consequently, social media plays a significant role in the success of mobile apps. 
Given this context, we have been trying to to evaluate the popularity spread of mobile apps by analyzing the social conversation on them. Twitter posts related to apps are an important segment of this conversation. However, when we tried to extract twitter posts related to specific apps we discovered that it was a difficult task, due, to the aliasing and name conflict problems. For instance when searching for tweets discussing the popular iPhone app titled "Movies by Flixster with Rotten Tomatoes - Free", we found that tweeters typically aliased this app simply as "Flixster". We then tried to simply search for tweets containing the term "Flixster". However, even this proved to be challenging as we discovered that "Flixster" is overloaded - it could refer to both the app or the website (http://www.flixster.com/) - its was not easy to discard the tweets referring to the website and retain those referring to the app. We found these issues to be common across many apps. Clearly, unless these issues are addressed meaningfully, it would be impossible to perform the core task, i.e., extracting tweets referring to apps.

In this paper, we present a strategy to reliably extract twitter posts that are related to specific apps, overcoming the aliasing and name conflict issues discussed above. While we were motivated by mobile apps, our techniques are completely general and may be applied to any entity class.

In the next section, we review related literature. In Section 3, we describe our solution approach. Section 4 experimentally demonstrates the efficacy of our techniques and in Section 6 we conclude the paper.

\section{Related Work}

The work related to this research may be classified as commercial or academic, and we discuss each in turn.

First let us address commercial solutions. Tweet filter [31] is a browser plugin that runs on top of twitter.com. Using Tweet filter we can filter tweets by matching usernames, keywords, phrases or source. Filter Tweets [9] is a browser based script for filtering tweets by a specific topic and it works only with the new Twitter version. One of the features in Filter Tweets is filtering tweets that contain a set of terms. Social Mention [26] is a social media search and analysis platform that aggregates user generated content from more than 100 social media web sites including: Twitter, Facebook, FriendFeed, YouTube, Digg, Google+ etc. It allows users to easily track and measure what people are saying about a person, company, product, or any topic across the web's social media landscape in real-time. Social Mention provides an API [27] to filter the user generated contents based on the given keywords from the popular social medias mentioned above.

All of the above-mentioned commercial solutions have similar characteristics. First, all of them work based on exact keyword match, however as described in the Section 1, mobile apps are seldom referred with the full name in the twitter posts, so it will be difficult, if not impossible, to find twitter posts related to mobile apps using any of three. In other words, these solutions do not address 
the aliasing or name conflict problems. We will demonstrate this experimentally in Section 4.

Let us now look at some academic research of relevance to our problem. Inherently, at the end, our aim is to classify each twitter post as whether it is related to a mobile app or not. Thus, at a high level our problem resembles a classification problem. In this respect the Bayesian classification technique is worth mentioning. The study titled "An Evaluation of Statistical Spam Filtering Techniques" 33] evaluates five supervised learning methods such as "Naive Bayes", "Maximum Entropy model", "Memory based learning", "Support vector machine" (SVM) and "Boosting" in the context of statistical spam filtering. They have studied the impact of different feature pruning methods and feature set sizes on each learner's performance using cost-sensitive measures. This study has observed that the significance of feature selection varies greatly from classifier to classifier. In particular,they found SVM, AdaBoost, and Maximum entropy model to be the top performers in this evaluation, sharing similar characteristics: not sensitive to feature selection strategy, easily scalable to very high feature dimension, and good performances across different data sets. In contrast, Naive Bayes [14|19], a commonly used classifier in spam filtering, is found to be sensitive to feature selection methods on small feature sets, and fails to function well in scenarios where false positives are penalized heavily. Many other studies [122 25] have studied the popularity of "Naive Bayes" [1419] in anti spam research and found that it outperforms keyword based filtering, even with very small training corpora.

The paper "Short Text Classification in Twitter to Improve Information Filtering" by Sriram et al. 29] has proposed an intuitive approach to determine the class labels and set of features with a focus on user intentions on Twitter. Their work classifies incoming tweets into categories such as News (N), Events (E), Opinions (O), Deals (D), and Private Messages (PM) based on the author information and features within the tweets. Their work is based on sets of features which are selected using a greedy strategy. Sriram et al.'s work experimentally shows that their classification out-performs the traditional "Bag-Of-Words" strategy. Unlike this resarch, our approach does not rely on supervised learning , thus we do not have the overhead of feature selection and manual labeling. In addition, we can classify a tweet as referring to any mobile app out of an arbitarily sized set of apps, unlike Sriram et al, who need a predefined exact number of categories into which they perform the classification.

In addition to classification of short text messages, integrating messages with meta-information from other information sources such as Wikipedia and WordNet 4112] are also relevant. Sankaranarayanan et al. 23] introduce TweetStand to classify tweets as news and non-news. Automatic text classification and hidden topic extraction [2914] approaches perform well, when there is meta-information or the context of the short text is extended with knowledge extracted using large collections. This does not apply in our case.

Currently, there are about 1 millions mobile apps in the market [18. To classify each twitter post as related to one or more of these apps, or not at all related to any of the mobile apps, will require equivalent number of classes, i.e. 1000,000 
classes in the classification approach. Such a large number of classes are impossible to handle using existing machine learning and classification techniques such as SVM [5] and Artifical Neural Networks(ANN) [8]. Therefore, instead of applying a classification approach, in this paper, we address the problem at hand using corpus based data driven approach. In the next section, we first describe the intuition behind our approach and then explain the algorithm in detail.

\section{Solution Approach}

We will first provide the intuition behind our approach and then delve into details. A precise statement of our problem is as follows: given app $\boldsymbol{A}$, find twitter posts that refer to $\boldsymbol{A}$. Our approach has two steps namely "Alias Identification" and "Conflict Resolution".

1. First we need to discover what alias is commonly used by users to refer to app A as names are often abbreviated in the length-restricted twitter posts (140 characters). For instance, the popular iTunes app "Doodle Jump - BE WARNED: Insanely Addictive", is commonly referred to in twitter posts as "Doodle Jump". We call this step the Alias Identification step.

2. After alias identification, we need to resolve name conflicts, i.e.,make sure that the twitter posts we find refer to the app and not to other objects with the same name. One particularly ripe area for conflicts is between mobile apps and a regular web applications. To see this consider the popular iPhone app titled "Movies by Flixster with Rotten Tomatoes - Free". It turns out that this app is commonly referred to as "Flixster". However, a twitter post containing the term "Flixster" might be referring to the app, or, to the highly popular sister website. We are of course interested in the popularity of the app. Similar issues arise in the case of the Facebook app, or the Google Translate app. We refer to this phase as Conflict Resolution.

\subsection{Intuition behind the Alias Identification Phase}

To identify the appropriate alias of an app with name $A$, we find the sub phrase contained in $A$ that is the most meaningful and unique. Such meaningfulness and uniqueness (described below) is judged in the context of a Social Media Corpus (SMC) we have constructed by lexical analysis of a vast amount of data gathered from Social Media Avenues such as Twitter, Facebook and the user comments awarded to apps in the native app stores.

Meaningfulness: Intuitively, meaningfulness refers to the semantic content of a phrase. For instance, in the context of the app title "Doodle Jump - BE WARNED: Insanely Addictive", the reader can easily see that the sub phrase "Doodle Jump" is more meaningful than, say "Be Warned", or "Insanely Addictive". From an information theoretic perspective, meaningful n-grams will exhibit higher collocation frequencies relative to individual occurrence frequencies of the constituent 1-grams. We describe this ratio as Affinity. Formally, we 
define the Affinity of a word phrase $P$ as, Affinity $(P)=\frac{f(P)}{\min _{\forall w_{i} \in P}\left(f\left(w_{i}\right)\right)}$, where $f(P)$ is the frequency of phrase $P$ in a corpus and $\min \left(f\left(w_{i}\right)\right)$ is the minimum frequency across the words in a phrase $P$ in the SMC. For the app name "Doodle Jump - BE WARNED: Insanely Addictive!", Table 1 shows the frequencies and affinity measurement of word phrases, which formally identifies the word phrase "Doodle Jump" as more meaningful than others. Note that the table does not show all phrases whose affinities are measured for comparison. For a particular $n(n=1 \ldots N$, where $\mathrm{N}$ is the number of words in the name of the application as the respective mobile app store), we take all $n$-grams from left to right beginning with the first word and stopping at the $(\mathrm{N}-\mathrm{n}+1)^{\text {th }}$ word.

Table 1. Affinity Measure

\begin{tabular}{|l|l|l|}
\hline Phrase & $f(P)$ & Affinity \\
\hline Doodle Jump & 99 & $99 / 1456=0.07$ \\
\hline Be Warned & 8231 & $8231 / 138408=0.06$ \\
\hline Insanely Addictive & 18 & $18 / 5315=0.003$ \\
\hline
\end{tabular}

Uniqueness: The meaningfulness property, while useful, is by itself not adequate for our purposes. To see this consider the following. Let us hypothetically assume (perhaps due to sampling biases while corpus creation) that the sub phrase "insanely addictive" is as (or more) meaningful than "Doodle Jump". Our system, using meaningfulness alone, would then judge "insanely addictive" as the best alias for the app "Doodle Jump - BE WARNED: Insanely Addictive" - a patently bad choice (as "insanely addictive" might be used in the context of many other apps). The uniqueness property (used in tandem with meaningfulness) prevents this mis-judgment, by ensuring that the selected alias is used often in the correct context, but rarely in alternate contexts. Furthermore, affinity does not apply to 1-grams and we cannot compare affinity directly to the uniqueness property we shall define. As such, this step will help to choose between the most meaningful n-gram phrase and all other 1-grams such that the end result is both highly meaningful and unique. Thus, to quantify uniqueness, we make a slight modification to the well-known IR notion of inverse document frequency (idf) [28] for a word or word phrase. The traditional idf is defined as: $i d f(P)=\log _{2} \frac{|D|}{1+d f(P)}$, where $|D|$ is the total number of documents in the corpus and $d f(P)$ is the document frequency of phrase $P$, namely the number of documents that contain phrase $P$ in corpus. We have modified this expression to: $i d f(P)=\log _{2} \frac{1}{1+\operatorname{tcount}(P)}$, where $\operatorname{tcount}(P)$ is the frequency of $P$ as recorded by Twitter in the target time interval $T$ and we have done away with $|D|$ because for all phrases, the number of documents in the corpus (in this case, number of tweets in Twitter's database) within the target time interval $T$ will be the same. Since we're looking for the highest $i d f(P)$ it does not matter what $|D|$ actually is. We retrieve phrase level tcount $(P)$ directly from Twitter. For instance, 
the $i d f$ of the phrase "Doodle Jump" in our corpus is 18.28 but the $i d f$ values of "Doodle" and "Jump" are 14.2 and 7.6 respectively. Therefore, "Doodle Jump" has more uniqueness and rarity than the individual terms "Doodle" and "Jump".

\subsection{Intuition behind the Conflict Resolution Phase}

The alias identification step ensures that the best alias is selected, but does not guarantee that this alias will not have conflicts with other object names, as the "Flixster" example above illustrated. In this phase we attempt to minimize this error. The core idea is as follows: Assume an alias, say $S$, is context-overloaded. Our objective is to identify the overloaded aliases and then rerun the core tweet search by using a new search term that consists of the alias and a few contextual terms that disambiguate the search(e.g., "flixster + iPhone"). The additional context raises the probability that the retrieved tweet is talking about the mobile app domain.

\subsection{Details of Alias Identification Phase}

As discussed in section 3.1, in this step we discover the alias $A^{\prime}$ of an app $A$, based on its meaningfulness and uniqueness values. This procedure is shown in Table 2 from steps 1-6. Here, step 1 extracts all sub phrases from $A$ (using a parser [2]), and computes affinities of each sub phrase in step 2. Subsequently, in step 3 we extract the most meaningful (highest affinity) phrase. This phrase is then subjected to a uniqueness test in step 4 by comparing its $i d f$ to the $i d f$ s of all 1-grams derived from $A$. Based on this test, the selected alias $A^{\prime}$ is returned.

After alias identification, the tweets containing this alias are considered $L e$ gitimate, while disqualified posts are marked as Spam. The legitimate tweets are then subjected to the conflict resolution phase, described below, to ensure that these refer to the app, and not to other objects with similar labels.

\subsection{Details of Conflict Resolution Phase}

To ensure that legitimate tweets refer to mobile apps and not to alternate objects, we design a classification mechanism where we first identify dual purpose aliases (e.g., Flixster, Facebook) and then incorporate additional context. More specifically, we run the $k$-means clustering algorithm [15] on all the $i d f$ values of the aliases $A^{\prime}$ with $k=2$, i.e. two clusters. The two initial mean points for each cluster are the lowest and the highest $i d f$ values across all aliases. This is shown in Table 2 in step 7 . The result of the $k$-means classification will be two sets of aliases, a high-idf cluster and a low-idf cluster. An example follows.

After partitioning the top ranked Android apps based on the $i d f$ values of their aliases, we found "paper toss", "pocket god", "words with friends", "ebay mobile", "pandora radio" and "espn scorecenter" belonged to the high- $i d f$ cluster, indicating they exist only in mobile app domain. Conversely, "flixster", "google earth", 
Table 2. Algorithm for retrieving exact query phrase to use on the tweet database to ensure high relevance

1. Generate set of all word phrases $\mathcal{C}$ of length 2,3 or 4 of the app name $A$. For example, for the app name "Doodle Jump - BE WARNED: Insanely Addictive!", some of the collocates will be "Doodle Jump", "Be Warned" and "Insanely Addictive".

2. Compute Affinity $\left(C_{i}\right)$ for each word phrase $C_{i} \in \mathcal{C}$ as derived in Step 1. For example, Af finity("Doodle Jump") = 0.07, Af finity("Doodle Jump Be") $=0.00068$ and Affinity ("Be Warned") $=0.06$.

3. Identify the word phrase $C_{i}^{\max }$ that has the highest value of $\operatorname{Affinity}\left(C_{i}\right)$. In our example, the highest value is for Affinity ("Doodle Jump") $=0.07$, thus $C_{i}^{\max }=$ "Doodle Jump".

4. Compute the $i d f$ for $C_{i}^{\max }$ and all one gram word of the name $A$. In our example, $i d f$ ("Doodle Jump") $=18.28$, idf("Doodle") $=14.2$, $i d f($ "Jump") $=7.6$, $i d f$ ("Warned") $=7.79$ and so on.

5. Identify the word phrase that has the highest $i d f$ as computed in step 4 . In example, "Doodle Jump" has the highest $i d f$.

6. Return the word phrase identified in Step 5 as the alternate app name $A^{\prime}$ of the app $A$.

7. After running steps 1-6 for all app names, we run k-means clustering on the $i d f$ values of the word phrases returned in step 6 with a $\mathrm{k}$ value of 2 and the initial means to be the highest $i d f$ and lowest $i d f$ values in the corpus respectively. This will yield two clusters, one that is high-idf and one that is low-idf.

8. For all word phrases that are part of the low-idf cluster, append extra context keywords before querying the tweet database. For all words phrases that are part of the high-idf cluster, we can use the word phrases "as is".

"skype" "facebook", "kindle", "bible", "flashlight", "netflix", "backgrounds" and "translator" are aliases with low $i d f$ values, indicating these names are used both in mobile apps and in other domains, such as web applications.

For the aliases with higher $i d f$, we accept their associated twitter posts, as there is a very high probability that the post is referring to the mobile app.

For the aliases with the low $i d f$ values, we incorporate additional filtering mechanisms, by adding additional keywords like "app", "Android", "iPhone", "iPod", "Apple" and "iPad". Tweets containing any of these additional keywords are considered relevant(Legitimate), otherwise it is categorized as Spam.

\section{Experimental Results}

In this section we demonstrate the efficacy of our approach, which we will refer to as TApp. The idea is to evaluate the quality of the legitimate tweets produced - if a tweet refers to the appropriate mobile app, the result is correct, otherwise, for that particular tweet, our procedure has failed. Specifically, we need to test for both Type 1 and Type 2 errors, i.e., how well we retain and how well we avoid the rejection of good tweets. First, we do a comparison with Naïve Bayesian approach. Next, we compare with one of the commercial platform, Socialmention [26]. 


\subsection{Comparison with Bayesian Approach}

For a baseline comparison, we have used Naïve Bayes classifier [14|19], a popular method for document classification in anti-spam research [1|22|25]. It is widely used in text categorization task [19] and often serves as baseline method for comparison with other approaches [33. In our implementation of Naïve Bayes (using the Laplacean prior to smooth the Bayesian estimation, as suggested in Nigam [19]) classification we extracted a set of keywords from every twitter post and used those as the feature set. Based on the key word occurrences in the twitter posts in the training data, probabilities are calculated. These probability values are used to classify the twitter posts.

Both the TApp and the Bayesian classification technique have been implemented using Java 1.6. We ran all the experiments using a Windows 7 machine with quad core processor of $2.33 \mathrm{GHz}$.

To compare TApp and the Bayesian classifier, we first selected a set of "apps of interest" - for this experiment, we chose the top 50 "hot" android apps using a popular mobile app search engine platform (http://www.appbrain.com/apps/ hot/). To create our test bed for these 50 apps, we randomly selected tweets from our database of 14 million tweets and manually verified whether they contained references to these apps (legitimate tweets) or not (spam). In this fashion we manually identified 1000 posts from our database, consisting of 500 posts that refer to one of these 50 apps (legitimate posts) and 500 tweets that refer to mobile apps or internet web sites, but not any of the selected 50 mobile apps. We apply both the Bayesian classifier and the TApp approach on this test bed to classify these 1000 posts into Legitimate and Spam. In Figures 1 and 2, we plot the histogram distributions of accuracy of the two approach - Bayesian and TApp. As can be seen from Figure 1 the Bayesian classifier identifies 337 out of the 500 Legitimate posts (a recall rate of $67 \%$ ), whereas the TApp approach demonstrates a recall of $97.2 \%$ by correctly classifying 486 of the 500 Legitimate posts. Similarly, as portrayed in Figure 2, the Bayesian classifier wrongly identified 174 of the 500 Spam posts as Legitimate, whereas TApp mis-identifies only 23 of 500. In Table 3 we have presented classical IR metrics such as precision, recall, true negative, accuracy and F-measure in both the cases. In all cases TApp significantly outperforms the Bayesian classifier (TApp scores above $90 \%$ in every case).

\subsection{Comparison with SocialMention}

SocialMention(SM) [26] is the leading social media search engine. To demonstrate the effectiveness of our approach, we decided to compare the accuracy of our results with those acquired from Socialmention. As discussed in the Section 2. the exact algorithms of Socialmention implementation is not known. However, by observing different search results we concluded Socialmention uses an exact keyword matching approach to identify the twitter posts that contains the given keywords. In this experiment, we used the same set of 50 apps used in the previous experiment in Section 4.1, For each app, we retrieved the tweeter 


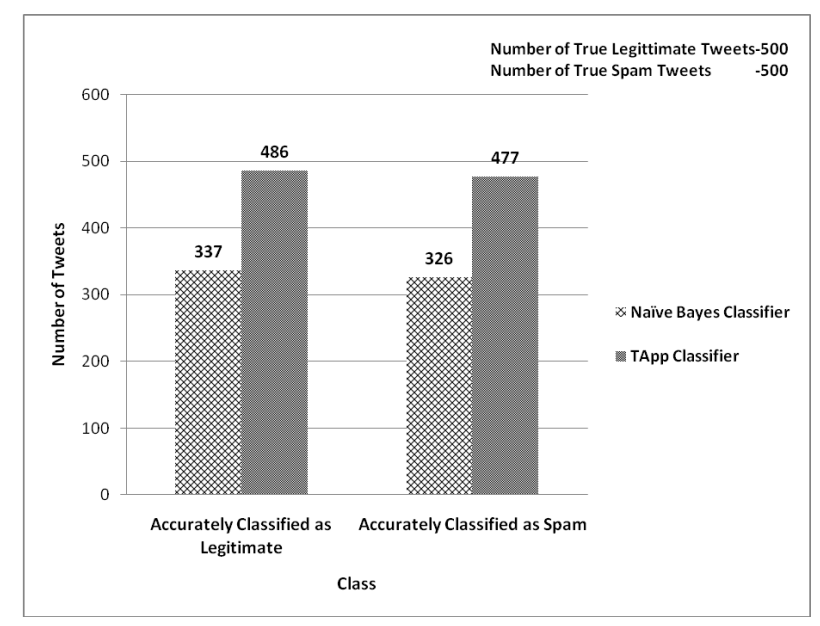

Fig. 1. Comparison of Accurate Classification

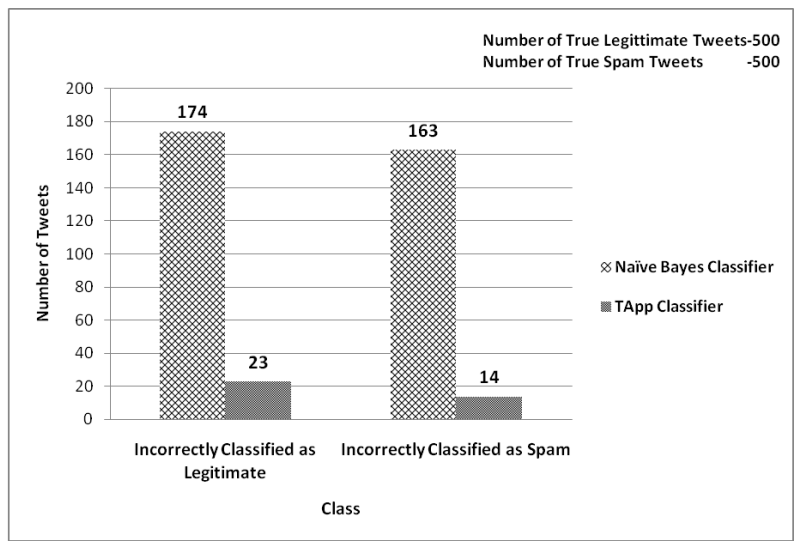

Fig. 2. Comparison of Incorrect Classification

Table 3. Comparison of IR metrics in Bayesian classifier vs. TApp

\begin{tabular}{|l|l|l|}
\hline Matrix & Naïve Bayes classifier & TApp classifier \\
\hline Precision & $100 * 337 /(511)=66 \%$ & $100 * 486 /(509)=95.6 \%$ \\
\hline Recall & $100 * 337 /(500)=67 \%$ & $100 * 486 /(500)=97.2 \%$ \\
\hline True Negative Rate & $100 * 326 /(500)=65.2 \%$ & $100 * 477 /(500)=95.4 \%$ \\
\hline Accuracy & $100 * 663 /(1000)=66.3 \%$ & $100 *(963) /(1000)=96.3 \%$ \\
\hline F-measure & $(2 * 65.9 * 67.4) /(66+67)=66.7 \%$ & $2 * 95.6 * 97.2 /(95.6+97.2)=96.4 \%$ \\
\hline
\end{tabular}


posts related to that app in the previous one month using both Socialmention API 27] and the TApp approach. The objective of our approach is to automate the Twitter post retrieval for large number of mobile apps. So, the input to both Socialmention and the TApp approach is app names as found in native app stores. The Socialmention uses these original app names to find the twitter posts. TApp approach applies name aliasing and name conflict resolution to retrieve the relevant tweets. However, the app names are chosen to be such that 22 out of 50 require either no aliasing and/or no name conflict resolution. This was done to access the effectiveness of the TApp technique in individually performing those 2 tasks.

To constrain the experimental data size, for each of the approach if the number of posts for an app is more than 50, we considered only the most recent 50 posts. Next, we passed the posts identified by both Socialmention and TApp along with the app names to two professional lexicographers. Each of the lexicographers has more than 5 years of experience of internet search optimization. They both worked together to arrive at an unanimous decision of which of these posts are "Valid" (i.e. the post is related to the respective app) and which of these are "invalid" (i.e. the post is not related to the respective app). We present the result in Table 4 .

As can be seen from Table 4, for many apps, the Socialmention platform has retrieved tweets that are not related to that app. In total only $43.44 \%$ of the total tweets retrieved by Socialmention has been identified as "Valid" post by lexicographers. Whereas, for TApp approach, the absolute number of invalid posts for each app is much smaller compared to the Socialmention. Overall $95.45 \%$ of the twitter posts retrieved by TApp has been identified as "Valid" by lexicograpghers. The total number of valid tweets retrieved by TApp is 1584 compared to 769 by Socialmention. So both in terms of accuracy and the coverage of retrieval, TApp significantly outperformed Socialmention.

Additionally, we observe that Socialmention works well in cases when there no aliasing of the app names and when there is no naming conflicts between the entity of interest and other objects. In these cases, Socialmention achieved $82.93 \%$ accuracy. For example, the extracted tweets for the apps "Live Holdem Poker Pro" "Google Sky Map", "Handcent SMS" and "Lookout Mobile Security" in both Socialmention and TApp are highly relevant because these names are only used in mobile app domain and there is no aliasing by users. One should observe that, even in these simple cases, where there is no name conflict and aliasing, the accuracy in TApp case is higher than that of Socialmention. The exact approach followed in Socialmention is unknown, so we are not sure of the reason behind this improvement, however we anticipate that this is due to the generic keyword matching algorithms followed in Socialmention, vs. the phrase search using tweeter API followed in TApp.

In the second scenario, when the app names required aliasing, but no name conflict resolution, the Socialmention's accuracy in retrieving relevant tweeter posts was $52.97 \%$ compared to $94.57 \%$ in TApp approach. For example, the tweets extracted for the apps "SpeechSynthesis Data Installer", "Kid Mode: 
Table 4. Comparison of Valid Tweets in Socialmention vs. TApp

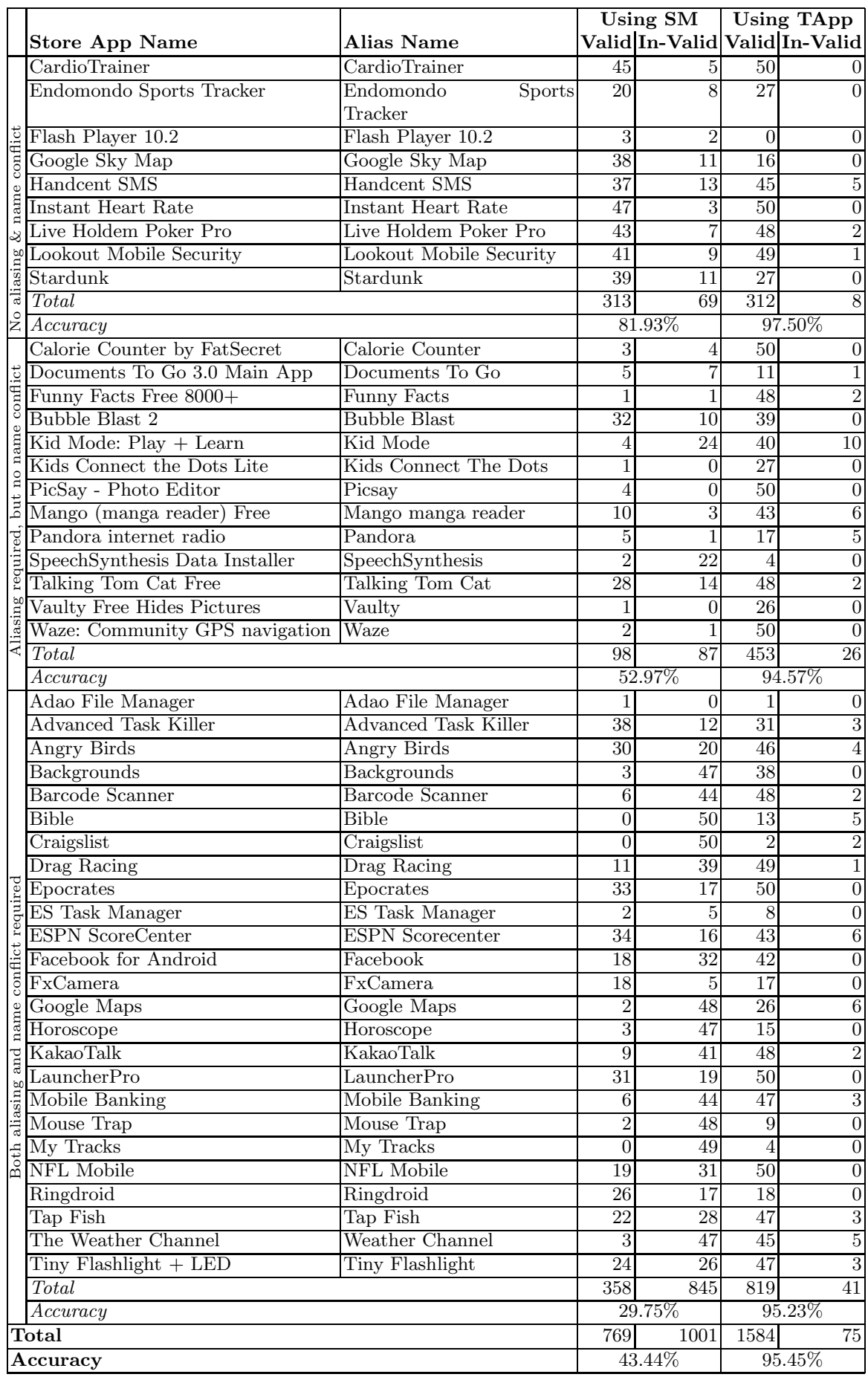


Play + Learn" and "Vaulty Free Hides" are mostly irrelevant or unfound because of aliasing practice of users when they post their tweets. These apps are typically referred to as "SpeechSynthesis" "Kid Mode" and "Vaulty" in most of the tweets.

To show the effectiveness of TApp's entity name conflict handling, we focus on the third category of app names, where both aliasing and name conflict resolution are required. If we look at the valid tweet count for the apps "Drag Racing", "Mouse Trap", "Mobile Banking" and "My Tracks" in case of Socialmention, they are very low compared to the invalid tweet count. These app names are used outside the mobile application domain as well and so required name conflict resolution in TApp approach, which is clearly not done in Socialmention. For these type of app names, Socialmention had a pretty low accuracy of just $29.75 \%$ in retrieving relevant tweets compared to $95.23 \%$ accuracy in TApp case.

This demonstrates the importance and effectiveness of both the aliasing and name conflict resolution steps in TApp.

\section{Discussion}

In this section we discuss the broader implications of our TApp approach. Our research falls in the design science research paradigm of Information Systems [35. We have developed an artifact that can successfully resolve name conflicts of app names in twitter posts. We have demonstrated the artifact through experimental study and a comparison with a manual method. Our two step approach out performs the benchmark Naïve Bayes classifier and a commercial implementation (Socialmention [26]) both on true negative and false positive errors.

Identifying social media mentions related to most popular products in general and mobile apps in particular has important implications for marketers as well as for product owners. Being able to predict the social media popularity of items have tremendous value to not only service providers but also marketers who would bid for ad-space on items with high potential popularity in order to maximize the exposure. TApp approach can be used to identify user generated contents across social media, which in turn can be used to measure product's popularity. Our approach can be utilized in many ICT research domains such as, identifying twitter posts related to brand monitoring in e-commerce, identifying the public opinions of e-participation, e-services and general e-government implementations by using the social media mentions, identifying students opinions of e-learning systems and analyzing the public views on digitizing the medical records of patients(Electronic Medical Records: EMR). Thus our approach is generalizable and broadly applicable across wide range of ICT research domains in general.

\section{Conclusion}

In this paper we have addressed the problem of reliably identifying tweets related to mobile apps. In the process we have addressed the aliasing and name conflict 
problems inherent in the task. We have compared the accuracy of our approach to Naïve Baysian approach and a commercial implementation (socialmention). Our approach outperformed in all measures of accuracy compared to Baysian approach and the socialmention. While our application is in the area of mobile apps, our techniques are generally applicable.

\section{References}

1. Androutsopoulos, I., Koutsias, J., Chandrinos, K.V., Ch, K.V., Paliouras, G., Spyropoulos, C.D.: An evaluation of naive bayesian anti-spam filtering, 9-17 (2000)

2. Apache, Open nlp, http://opennlp.sourceforge.net/projects.html (last accessed July, 2012)

3. AppleiOS. Apple-ios, http://www .apple.com/ios/ (last accessed on July 14, 2011)

4. Banerjee, S.: Clustering short texts using wikipedia. In: Proceedings of the 30th Annual International ACM SIGIR Conference (2007)

5. Chang, C.C., Lin, C.J.: LIBSVM: a library for support vector machines (2001)

6. Cui, H., Wen, J.-R., Nie, J.-Y., Ma, W.-Y.: Query expansion by mining user logs. IEEE Transactions on Knowledge and Data Engineering 15(4), 829-839 (2003)

7. Dent, K., Paul, S.: Through the twitter glass: Detecting questions in micro-text. In: Proceedings of AAAI 2011 Workshop on Analyzing Microtext (2011)

8. Fausett, L. (ed.): Fundamentals of neural networks: architectures, algorithms, and applications. Prentice-Hall, Inc., Upper Saddle River (1994)

9. Filtertweets, Filter tweets for greasemonkey, http://userscripts.org/scripts/ show/87289 (last accessed June 27, 2012)

10. Google Inc. Android developers, http://developer.android.com/index.html (last accessed on July 14, 2011)

11. Google Inc. Google search appliance help center, http://code.google.com/ apis/searchappliance/documentation/46/help_gsa/serve_synonym.html (last accessed on May 13, 2011)

12. Hu, X., Sun, N., Zhang, C., Chua, T.-S.: Exploiting internal and external semantics for the clustering of short texts using world knowledge. In: CIKM 2009: Proceeding of the 18th ACM Conference on Information and Knowledge Management, pp. 919-928. ACM, New York (2009)

13. Jansen, B.J., Liu, Z., Weaver, C., Campbell, G., Gregg, M.: Real time search on the web: Queries, topics, and economic value. Inf. Process. Manage. 47, 491-506 (2011)

14. Lewis, D.D.: Naive (bayes) at forty: The independence assumption in information retrieval, pp. 4-15. Springer (1998)

15. MacQueen, J.B.: Some methods for classification and analysis of multivariate observations. In: Cam, L.M.L., Neyman, J. (eds.) Proc. of the fifth Berkeley Symposium on Mathematical Statistics and Probability, vol. 1, pp. 281-297. University of California Press (1967)

16. Markets \& Markets. World mobile applications market - advanced technologies, global forecast (2010-2015), http://www.marketsandmarkets.com/ Market-Reports/mobile-applications-228.html (last accessed on May 13, 2011)

17. Mashable. Mobile app market to surge to $\$ 17.5$ billion by 2012 , http://mashable.com/2010/03/17/mobile-app-market-17-5-billion/

(last accessed on May 13, 2011) 
18. Mobilewalla. Mobilewalla-an app search engine, http://mobilewalla.com/ (last accessed on May 13, 2012)

19. Nigam, K.: Using maximum entropy for text classification. In: IJCAI 1999 Workshop on Machine Learning for Information Filtering, pp. 61-67 (1999)

20. One Riot. The inner workings of a realtime search engine (2009)

21. PC World. It's android vs. apple: Will you switch sides?, http://www.pcworld.com/article/199109/its_android_vs_apple_will_you_ switch_sides.html (last accessed on May 13, 2011)

22. Sahami, M., Dumais, S., Heckerman, D., Horvitz, E.: A bayesian approach to filtering junk E-mail. In: Learning for Text Categorization: Papers from the 1998 Workshop, Madison, Wisconsin, AAAI Technical Report WS-98-05 (1998)

23. Sankaranarayanan, J., Samet, H., Teitler, B.E., Lieberman, M.D., Sperling, J.: Twitterstand: news in tweets. In: Proceedings of the 17th ACM SIGSPATIAL International Conference on Advances in Geographic Information Systems, GIS 2009, pp. 42-51. ACM, New York (2009)

24. Sarkas, N., Bansal, N., Das, G., Koudas, N.: Measure-driven keyword-query expansion. Proceedings of the Vldb Endowment 2, 121-132 (2009)

25. Schneider, K.-M.: A comparison of event models for naive bayes anti-spam e-mail filtering. In: Proceedings of the 11th Conference of the European Chapter of the Association for Computational Linguistics (EACL 2003), pp. 307-314 (2003)

26. SocialMention. Social mention, http://socialmention.com (last accessed on November 5, 2011)

27. SocialMention. Social mention api, http://socialmention.com/api/ (last accessed on November 5, 201)

28. Spärck Jones, K.: A statistical interpretation of term specificity and its application in retrieval. Journal of Documentation 28(1), 11-21 (1972)

29. Sriram, B., Fuhry, D., Demir, E., Ferhatosmanoglu, H., Demirbas, M.: Short text classification in twitter to improve information filtering. In: Proceeding of the 33rd International ACM SIGIR Conference on Research and Development in Information Retrieval, SIGIR 2010, pp. 841-842. ACM, New York (2010)

30. Techcrunch. Report: Mobile app market will be worth $\$ 25$ billion by 2015 apple ios share: 20\%, http://techcrunch.com/2011/01/18/report-mobile-appmarket-will-be-worth-25-billion-by-2015-apples-share-20 (last accessed on May 13, 2011)

31. TweetFilter. Tweetfilter for greasemonkey, http://userscripts.org/scripts/ show/49905 (last accessed June 26, 2012)

32. Venture Beat. Why apple can not beat android, http://venturebeat.com/ 2010/11/05/why-apple-cant-beat-android (last accessed on May 13, 2011)

33. Zhang, L., Zhu, J., Yao, T.: An evaluation of statistical spam filtering techniques. ACM Transactions on Asian Language Information Processing (TALIP) 3 (2004)

34. Znet. Android vs. apple: The 2011 cage match, http://www.zdnet.com/blog/ btl/android-vs-apple-the-2011-cage-match/43682 (last accessed on May 13, 2011)

35. Hevner, A.R., March, S.T., Park, J., Ram, S.: Design science in information systems research. MIS Quarterly 28(1), 75-105 (2004) 